\title{
Das kulturelle Gedächtnis
}

\author{
Jan Assmann
}

Zusammenfassung: Kultur ist Gedächtnis: diese These wird eingangs anhand der mnemotechnischen und identitätsstabilisierenden Funktion von Formgebung allgemein erläutert und dann am Beispiel geformter Sprache näher ausgeführt. Ausgehend vom Textbegriff K. Ehlichs, der „Text“ als "wiederaufgenommene Mitteilung" bestimmt im Rahmen einer ,zerdehnten Situation“, wird Kultur als der Oberbegriff aller zerdehnten Situationen verstanden, in der „kulturelle Texte“ immer wieder aufgenommen werden und so eine sich fallweise über Jahrtausende erstreckende ,zerdehnte Situation" diachroner Kommunikation fundieren. So bilden sich spezifische Zeithorizonte heraus, die von Gesellschaften als die ihnen eigene Vergangenheit beansprucht und bewohnt bzw. „erinnert“ werden. Dieses kulturelle Gedächtnis wird mit wachsendem Schriftgebrauch komplex, weil sich um den Zentralbestand kultureller Texte herum immer weiter ausgreifende Peripherien nicht mehr gebrauchter, aber vor dem Verschwinden bewahrter Texte ablagern, wodurch eine Dynamik des Vergessens und Erinnerns in Gang kommt. Diese Dynamik steigert sich, wo eine Gesellschaft sich von ihrer Vergangenheit abkehrt und die mit ihr verbundenen kuturellen Texte nicht einfach "vergißt“, sondern „verdrängt“, wie es in paradigmatischer Weise bei der Entstehung des christlichen Abendlandes in Abkehr vom antiken Heidentum geschah.

Summary: Culture is memory: this thesis highlights the mnemotechnical aspects and functions of cultural ",morphology" in general and of formed speech in particular. On the basis of K. Ehlich's notion of text as „reproduced communication“ in the frame of an „extended situation“, culture is interpreted as the totality of extended situations, in which „cultural texts“ are continually reproduced and an ,extended situation" is institutionalized that may extend over several millennia. In this way, specific temporal horizons are formed which given societies claim and remember as "their" past. This form of cultural memory grows complex in the process of growing literacy. Around a core of highly normative and formative cultural texts, an ever growing periphery of texts is developing which fell out of use but are still preserved in written form. This dynamic of forgetting and remembering becomes dramatic in cases when a society rejects its past altogether and not only "forgets" but suppresses its cultural texts as was the case when emerging Christianity rejected pagan antiquity.

\section{Form und Prägnanz als mnemotechnische Verfahren}

((1)) Stellen wir uns den Menschen als ein Lebewesen vor, das wie die Tiere in der Zeit lebt, wächst, reift und altert, fortwährend in der Zeit agieren und reagieren muß, zugleich aber, anders als die Tiere, ein Bewußtsein und Gedächtnis ausbildet, das den jeweiligen Zeitpunkt seines Handelns und Reagierens einbettet in einen weiten Horizont von Erinnerung und Erwartung, Wissen und Planen, und gehen wir weiter davon aus, daß diese den Augenblick weit übergreifende Innenwelt von Bewußtsein und Gedächtnis sich im Umgang mit anderen entwickelt, im Zuge seiner Sozialisation und Kommunikation, und daher ebensosehr eine Sache der sozialen Zwischenwelt wie der individuellen Innenwelt ist: Dann verstehen wir, daß dem Menschen, sowohl als Individuum als auch als Gruppe, alles daran gelegen sein muß, diese geistige Innen- und Zwischenwelt im Fluß der Zeit zu stabilisieren. Gerade dieser unsichtbare Erinnerungs- und Erwartungs-, Wissens- und Erfahrungsraum, in dem er sich als Einzelner und als Gruppe orientiert, muß ihm als das Flüchtigste erscheinen, das es vor allen anderen Dingen sicherzustellen und dauerhaft zu machen gilt. Der Mensch lebt daher immer schon, soweit wir ihn zurückverfolgen können, in einer von Merkzeichen erfüllten Welt, die um so reicher und komplexer wird, je größer und komplexer die Gruppen, Gemeinschaften und Gesellschaften werden, in denen er lebt. Diese Merkzeichen erschließen, symbolisieren, vergegenwärtigen ihm die "Welt", in die seine
"Umwelt" eingebettet ist, und machen ihn zu einem Wesen, das, in Nietzsches Worten, nicht wie die Tiere "an den Pflock des Augenblicks gekettet” ist, sondern sich flexibel in größeren Zusammenhängen zu orientieren und sogar über den eigenen Tod hinaus zu denken imstande ist. Diese Welt von Merkzeichen können wir "Kultur" nennen und wir können sie sogar als eine "Mnemotechnik" verstehen, insofern sie darauf angelegt ist, der geistigen Innen- und Zwischenwelt Stabilität und Permanenz zu verleihen und sie der Flüchtigkeit und Vergänglichkeit des Lebendigen zu entreißen.

((2)) Der Drang zur Stabilisierung manifestiert sich in der Dingwelt, mit der sich die Menschen umgeben, als Wille zur Form. Die Formenwelt der Werkzeuge und Waffen, Geräte, Keramik, Bilder und Bauten, die als materielle Spuren menschlicher Tätigkeit sich über Jahrtausende und Jahrzehntausende zurückverfolgen lassen, ist von einer strikten Regelmäßigkeit durchwaltet, die sie nicht anders als die Formenwelt der Natur einer morphologischen Analyse zugänglich und das einzelne Objekt in seiner zeitlichen und räumlichen Zugehörigkeit bestimmbar macht. ${ }^{1}$ Die Bestimmbarkeit des Objekts verdankt sich seiner morphologischen Prägnanz, und diese ist wiederum Ausdruck jenes Willens zur Form, den wir als Ausdruck einer Gedächtnisleistung oder Traditionsbildung bestimmt haben. Die Form wird nicht immer wieder neu erfunden, sondern steht in einer Tradition, die sie voraussetzt und aufnimmt, auch dort, wo sie sie willentlich verändert und 
abwandelt. Wäre es anders, gäbe es keine Ur- und Frühgeschichte, deren morphologische Methodologie sich in alle anderen Kulturwissenschaften hinein fortsetzt, die es mit späteren und komplexeren Formenwelten zu tun haben - Altertums-, Kunst-, Musik-, Literaturwissenschaften usw. spiegeln in ihrer Methodologie die morphologische Prägnanz der Kultur, die auf ihre Gedächtnisförmigkeit verweist, auf ihren Aspekt einer Mnemotechnik, durch den sich die spezifischen Merkmale der Zeit und des Raumes, des individuellen und des kollektiven Stils unverkennbar in das Objekt einschreiben.

((3)) Der Wille zur Form prägt sich nicht nur den Objekten ein, sondern auch dem Handeln selbst, und zwar desto stärker, je mehr auch diese Handlungen als Merkzeichen gedacht sind, die über die Zeit ihres Vollzugs hinaus wirksam bleiben sollen, etwa indem sie bestimmte Verhältnisse verbindlich auf Dauer stellen, ein für alle Mal gültige Absprachen besiegeln, langfristige Erwartungen sichern sollen. Solche geformten Handlungen nennen wir Riten. ${ }^{2}$ Sie gehen über bloße Routinen hinaus in dem Maße, wie die Formung nicht einfach das Handeln entlasten, sondern im Sinne der Merkzeichenfunktion einen Sinngehalt auf Dauer stabilisieren soll, der im primären Zweck der Handlung nicht aufgeht. Das Mähen des Getreides z.B. ist eine Handlung, die in ihrem technischen Ablauf stark formalisiert ist, ohne darum ein Ritus zu sein. Sie bezweckt nichts anderes als einen wichtigen Schritt im Zusammenhang der Getreideernte. Wenn aber das Abmähen der ersten Ähren feierlich begangen wird, z.B. mit dem Ziel, dadurch die Fülle dieser und aller folgenden Ernten sicherzustellen, wie etwa im altägyptischen Minfest, dann sprechen wir von einem Ritus. Anders als in der Objektwelt, in der der Faustkeil, die Pfeilspitze, der Topf und der Haustyp trotz aller morphologischen Prägnanz nichts anderes bezwecken und auf nichts anderes verweisen als die jeweilige Alltagsfunktion, Werkzeug, Waffe, Gefäß und Behausung zu sein, verweisen die Riten per definitionem über den Horizont des Alltags und der alltäglichen Handlungsziele hinaus auf eine höhere, allgemeinere, übergeordnete Sphäre, die in ihnen in der Art von Symbolen zur Erscheinung kommt. Wir können im Hinblick auf die Riten und die in ihnen greifbaren Ausprägungen eines Willens zur Form von einer doppelten Ebene der Formung sprechen: einer praktischen und einer symbolischen. Auf der praktischen Ebene geht es z.B. um das Essen und Trinken von Brot und Wein, auf der symbolischen Ebene dagegen um das Gedenken an den Kreuzestod Christi und die Teilhabe an seiner Heilswirkung. Dieser Ritus wurde mit den Worten "solches tut zu meinem Gedächtnis" eingesetzt, aber auch wo diese mnemotechnische Funktion nicht explizit herausgestellt wird, haben wir es mit Merkzeichen, hebräisch zikkaron, zu tun. Jeder Ritus ist ein zikkaron, entweder an ein historisches oder historisch gemeintes Ereignis wie der Auszug aus Ägypten und der Kreuzestod Christi, oder an ein mythisches Ereignis wie die Geburt der Isis oder der siebte Schöpfungstag.

((4)) Anders also als bei den Objekten tritt bei den Handlungen noch etwas hinzu, um sie in ihrer stabilisierenden Merkzeichenfunktion auszuzeichnen und sie aus der Sphäre der Alltagszwecke herauszuheben. Wenn wir uns mit dieser Beobachtung zur Sphäre der Objekte zurückwenden, stellen wir jedoch sehr bald fest, daß auch bei ihnen etwas hinzutritt. Die Kanne erfüllt ihren Alltagszweck nicht besser, wenn sie mit Ornamenten oder Figuren dekoriert ist. Sie gewinnt aber an formaler Prägnanz. Die Merkmale, die sie für den Archäologen bestimmbar machen, vermehren sich in zeitlicher, räumlicher, sozialer und funktionaler Hinsicht. Das dekorierte Objekt trägt diese Merkmale nicht nur wie Symptome an sich, die dem Kenner seine Herkunft verraten, sondern trägt sie auch als Symbole zur Schau, die eine Botschaft vermitteln wollen. Ähnlich wie bei den Riten wird bei den Artefakten die Ebene der Funktionalität oder Pragmatik überformt durch eine zweite Ebene der Symbolizität oder Semantik. Diese Ebene bildet den Ursprung des Ästhetischen, auch wenn der Wille zur Form, der sich hier als Wille zum Dekor manifestiert, zunächst nicht auf "interesseloses Wohlgefallen" abzielt, sondern, ähnlich wie bei den zu Riten gesteigerten Handlungen, auf die Sichtbarmachung eines übergreifenden, verbindenden und der Flüchtigkeit zu entreißenden, im Medium der Form zu stabilisierenden Wissens.

((5)) Die Unterscheidung zwischen Pragmatik und Semantik wird in einem ganz anderen Sinne entscheidend, wenn wir uns nun nach den Handlungen und Objekten der Sprache zuwenden. Sprache nennen wir die Erzeugung von Lauten zum Zwecke der Bezeichnung von Sinneinheiten. Die Ebene der "Symbolizität" ist hier also bereits primär und irreduzibel gegeben. Sprache ist die Erzeugung von Symbolen. Die Symbolizität der Sprache verdankt sich nicht erst der Formung, sondern bereits der Artikulation. Das gilt so weder für Objekte noch für Handlungen. Daher ist die Sprache immer schon und vor aller spezifischen Formgebung Gedächtnis, d.h. ein Speicher kollektiver Erfahrung und Orientierung, der zugleich mit ihrer Erlernung erworben wird. Die Sprache beruht auf dem Prinzip der doppelten Artikulation. Auf der Ebene der Lauterzeugung artikuliert sie sich in einer geschlossenen und relativ kleinen Menge distinkter Phoneme und auf der Ebene der Sinnverweisung in einer großen und innerhalb gewisser Grenzen flexiblen Menge von Semen und Sememen. Das Prinzip der doppelten Artikulation ist der Sprache eigentümlich und gilt so für keine andere Form menschlicher Objekte und Handlungen. Man kann sie in anderen Medien nachahmen wie z.B. in der Pantomime, der Programm-Musik oder der Bilderzählung, aber das heißt nur, diese Medien als eine Art von Sprache zu verwenden, so wie wir ja auch von Gebärdensprache, Formensprache, "Klangrede" (Harnoncourt) sprechen. Artikulation ist jedoch etwas anderes als Formung. Es entreißt Sprachhandlungen um nichts der Flüchtigkeit und Vergänglichkeit, ob sie nun stärker oder schwächer artikuliert sind; das trägt allenfalls zu ihrer Verständlichkeit, d.h. ihrem kommunikativen Funktionieren bei. Sollen sie darüberhinaus erhalten bleiben, weiter wirken, d.h. zu Merkzeichen werden, dann müssen sie über die Artikulation hinaus geformt werden. Erst formale Prägnanz verleiht ihnen die Stabilität kultureller Merkzeichen. Erst durch Formung erwirbt eine sprachliche Äußerung die Merkmale, die sie einerseits einreihen in eine Tradition sprachlicher Formung und dadurch zu einem Gedächtnisträger machen und andererseits erinnerbar machen für spätere Rückgriffe, so daß sie dieses Gedächtnis zugleich voraussetzt und bereichert.

((6)) So finden wir die beiden Ebenen, die wir bei den Objekten und den Handlungen unterschieden haben, Pragmatik und Semantik, auch bei der Sprache wieder. Wir müssen sie hier 
nur anders nennen, denn die Semantik ist hier aufgrund des Prinzips der doppelten Artikulation bereits der Ebene der Pragmatik eingeschrieben. Ohne Semantik würde Sprache nicht funktionieren, d.h. nicht Sprache sein. Hier ist Semantik bereits Gegenstand der Artikulation, nicht erst der Formung. Was kommt hier durch Formung hinzu? Form wirkt auch hier als Stabilisator. Sie hat keinen anderen Sinn, als Semantik haltbar zu machen. Eric Havelock nannte das "preserved communication", Niklas Luhmann prägte dafür, unter Verweis auf Havelock, den Begriff der "gepflegten Semantik". ${ }^{3}$ In unserer Terminologie wird eine sprachliche Äußerung durch das Hinzutreten der Formung zum "Text". Text, im ursprünglichen, landläufigen und auch in einem bestimmten linguistischen Verständnis, auf das ich gleich eingehen werde, bedeutet "geformte Sprachäußerung", und zwar im Hinblick auf Erinnerung, Überlieferung und Wiederaufnahme geformte sprachliche Äußerung. Text ist Sprache im Status des Merkzeichens. Mit der Formung überschreiten wir, in unserer Terminologie, die Grenze zwischen dem kommunikativen zum kollektiven und kulturellen Gedächtnis.

((7)) Daher gelten die Gesetze der Morphologie für die Sprache genauso wie für alle anderen "Merkzeichen" der menschlichen Dingwelt. Auch hier äußert sich in den zu Merkzeichen bestimmten Äußerungen ein Wille zur Form, der das Wort durch Formung über den Augenblick seiner Verlautbarung hinaus zu stabilisieren versucht. Reim, Assonanz, Parallelismus membrorum, Allitteration, Metrum, Rhythmus, Melos sind Verfahren solcher Stabilisierung, die dem Flüchtigen Dauer verleihen sollen im Strom der Zeit und es dem morphologisch geschulten Blick bestimmbar machen in Raum und Zeit, nach Gattung und "Sitz im Leben", Funktion und Tradition.

\section{Text und "zerdehnte Situation"}

((8)) Was ist ein Text? Diese Frage kann man auf zwei Weisen beantworten. Man kann erstens nach der Herkunft des Wortes fragen, um seine Bedeutung von seiner ursprünglichen Bedeutung her zu erschließen, und man kann zweitens dem gegenwärtigen Alltagsgebrauch des Wortes nachgehen, um den Umkreis der damit bezeichneten Phänomene zu bestimmen. Was die Herkunft angeht, werden wir auf das lateinische Wort textus verwiesen, das von texere "weben" abgeleitet ist und "Gewebe" bedeutet. Von dieser Metapher ausgehend verwendet Quintilian den Begriff textus für den Zusammenhang der Worte (verborum), Aufbau und Kohärenz der Rede. Wir wollen diesen Textbegriff den rhetorischen nennen.

((9)) Die Rhetorik unterscheidet zwischen Information und Mitteilung, Sachverhalt und "Sprachverhalt". Diese Unterscheidung war den alten Kulturen bis dahin fremd geblieben. Das ägyptische $m d t$ und das hebräische dabar bezeichnen unterschiedslos das Wort und die im Wort zur Sprache gebrachte Sache, Angelegenheit. Wer sich auf ein Gesagtes bezieht, kann nicht zum Ausdruck bringen, ob er Form oder Inhalt des Gesagten meint. Zwischen Rede und Sache ("Mitteilung" und "Information" in der Terminologie N. Luhmanns) besteht kein Unterschied. Genau auf diesen Unterschied kommt es aber der Rhetorik an, und aus diesem Interesse heraus entwickelt sie den Begriff des Textes, der sich präzise auf den Sprachverhalt, nicht auf den Sachverhalt bezieht.

((10)) Später übernimmt die Philologie von der Rhetorik den neuen Begriff textus und wendet ihn auf das Objekt ihrer Arbeit an. Textus steht hier in Opposition zu commentarius. Textus nennt man jene sprachliche Äußerung, auf die man sich in der Form des commentarius bezieht, commentarius dagegen jene Äußerungsform, die einen textus zum Gegenstand hat. Zum Text wird eine sprachliche Äußerung als Objekt philologischer Arbeit: Textkritik, Herstellung des Textes, Edition, Kommentierung, auch: Übersetzung. Der Begriff Text hat also im Primärhorizont sprachlicher Kommunikation zunächst einmal keinerlei natürliche Evidenz. Von Texten spricht man erst im Sekundärhorizont einer philologischen Auslegungskultur. Daher ist der Begriff den meisten alten Sprachen unbekannt. Er kommt erst im Kontext von Rhetorik und Philologie, d.h. metatextueller Reflexion auf. "Text", in anderen Worten, ist ein Begriff der Metasprache, nicht der Objektsprache.

((11)) Im alltäglichen Gebrauch verstehen wir unter "Text" im allgemeinen ein Schriftstück. In der Sprachwissenschaft, insbesondere der Textlinguistik, wurde dieser Bezug auf die Schriftform aufgegeben. Hier nennt man "Text" zum einen die oberste sprachliche Einheit oberhalb des Satzes und zum anderen die natürliche und konkrete Form sprachlicher Äußerungen. Um es mit dem Linguisten Peter Hartmann, einem der Väter der modernen Textlinguistik, zu sagen: „,Es wird, wenn überhaupt gesprochen wird, nur in Texten gesprochen“. ${ }^{4}$ Text ist die Form, in der Sprache in der Natur vorkommt, alle anderen Einheiten wie Satz, Satzglied, Wort, Morphem sind artefizielle Zergliederungen, analytische Konstrukte.

((12)) Dieser Textbegriff steht jedoch im Widerspruch zum alltäglichen Sprachgebrauch, in dem immer noch etwas von der ursprünglichen Bedeutung des Wortes textus mitschwingt. Es ist das Verdienst des Linguisten Konrad Ehlich, dieses entscheidende semantische Element in einem neugefaßten sprachwissenschaftlichen Textbegriff sinnvoll aufgehoben zu haben. ${ }^{5}$ Ehlich definiert Text als ,wiederaufgenommene Mitteilung“. Die Urform des Textes ist bei ihm nicht das kommentierte Werk, sondern die ausgerichtete Botschaft; evident ist aber der gemeinsame Nenner: die Wiederaufnahme. Entscheidend für die Genese von Texten ist die Ablösung von der unmittelbaren Sprechsituation. Damit ist, nach Ehlich, erstens die Kopräsenz von Sprecher und Hörer nicht mehr gegeben, und wird zweitens die akustische Dimension als Übertragungsmittel für den Äußerungsakt problematisch. Die Ablösung von der unmittelbaren Sprechsituation wird dann unausweichlich, wenn der Sprecher räumliche und/oder zeitliche Distanzen überwinden muß, um den Hörer zu erreichen. $\mathrm{Zu}$ diesem Zweck muß die Sprechhandlung über die unmittelbare Situation hinaus aufbewahrt werden, um in eine zweite Sprechsituation hineintransportiert zu werden. Ehlich erläutert das am Beispiel des Boten, der die Botschaft auswendig lernt, um sie anderenorts vor dem intendierten Hörer reproduzieren zu können. Hier können wir mit vollem Recht vom "Text" der Botschaft sprechen. Nicht die Schriftform ist entscheidend, sondern der Akt der Speicherung und Überlieferung. 
Die beiden Situationen, Sprecher und Bote sowie Bote und Hörer, sind in Raum und Zeit getrennt und doch durch den Text und den Prozeß seiner Überlieferung miteinander vermittelt. An die Stelle der einen unmittelbaren Situation der Kopräsenz tritt die "zerdehnte Situation", die sich in zwei bis virtuell unendlich viele einzelne Situationen entfalten kann und deren Grenzen nur durch die Vorfindlichkeit des Textes und den Prozeß seiner Überlieferung bestimmt werden.

((13)) Aus der ursprünglichen Korrelation der Begriffe textus und commentarius ist die Korrelation der Begriffe Text und Überlieferung geworden. Texte sind Sprechakte im Kontext zerdehnter Situationen. Dieser Textbegriff hat zwei Vorteile. Einerseits kommt er los von der alltagssprachlichen, theoretisch unerwünschten Bindung an Schriftlichkeit und erlaubt den Begriff des mündlichen Textes. Andererseits greift er durch seine Bindung an den Begriff der Überlieferung ein zentrales Bedeutungselement der philologischen Tradition wieder auf. Text ist nicht jede, sondern nur diejenige sprachliche Äußerung, mit der sich auf Seiten des Sprechers ein Bedürfnis nach Überlieferung und auf Seiten des Hörers ein Bedürfnis nach Wiederaufnahme verbindet, also Äußerung, die auf eine Art von räumlicher und/oder zeitlicher Fernwirkung hin angelegt ist und auf die man über die Distanz hinweg zurückgreift. Darin schwingt noch etwas von dem Adelstitel mit, den das Wort textus einmal bezeichnete. Hier sind Bindekräfte am Werk, die nicht nur die einzelnen Wörter zum "Gewebe" des Textes verbinden, sondern Sprecher und Hörer über raumzeitliche Grenzen hinweg miteinander zu verbinden vermögen, und die daher mit dem Begriff der "Verbindlichkeit" nicht unangemessen bezeichnet werden. Texte sind Äußerungen gesteigerter Verbindlichkeit.

((14)) Der Begriff der "zerdehnten Situation" umfaßt sowohl die Überlieferung als auch die Wiederaufnahme von Texten. Zerdehnte Situationen kommen in der Natur nicht vor und müssen kulturell institutionalisiert werden. Sie können sich nicht entwickeln und erhalten ohne institutionelle Stützung und Rahmung. Das gilt schon für das Institut des Boten. Es muß sichergestellt werden, daß der Bote die Botschaft korrekt erinnert, daß er seinen Bestimmungsort erreicht und daß der Empfänger ihn als den Vertreter des Senders anerkennt: haššălu $u^{a}$ kaššole ${ }^{a} h$ 'oto "Der Bote ist wie der, der ihn sendet" wie es das jüdische Boteninstitut bestimmt. ${ }^{6}$

((15)) Die "zerdehnte Situation" läßt sich als die ursprünglichste Lösung des Problems verstehen, von dem wir ausgegangen sind: dem Bedürfnis nach Stabilisierung in einer flüchtigen Welt. Wenn Kulturen, wie Thomas Macho einmal in einem Rundfunkinterview sagte, "Inseln im Meer des Vergessens" sind, dann ist die "zerdehnte Situation" die Urszene der Kultur. Hier wird etwas der Flüchtigkeit der Kommunikation und dem Meer des Vergessens entrissen, um es für den Fall einer späteren Wiederaufnahme zu bewahren.

\section{Horizontbildung}

((16)) Die Kultur läßt sich als die umfassendste aller zerdehnten Situationen begreifen, als der Inbegriff aller zur Wiederaufnahme bestimmter Botschaften und aller Institutionen, derer es bedarf, um diese Prozesse der Formulierung, Überlieferung, Zirkulation und Wiederaufnahme zu stabilisieren. Als die "zerdehnte Situation" schlechthin schafft die Kultur einen weit in die Vergangenheit ausgreifenden Horizont eigener Zeitlichkeit, in der das Vergangene präsent bleibt und eine eigentümliche Form von Gleichzeitigkeit herrscht, so daß Homer und Shakespeare, Platon und Dante, Vergil und Goethe nebeneinander zu stehen kommen und wir uns als Empfänger ihrer Botschaften fühlen können.

((17)) Mit dem kulturellen Gedächtnis erschließen sich vieltausendjährige Erinnerungsräume, und es ist die Schrift, die dabei die entscheidende Rolle spielt. Das läßt sich schon im Altertum beobachten. Im 1. Jahrtausend v. Chr., im Anschluß an den Untergang der bronzezeitlichen Hochkulturen, entwikkelten sich in verschiedenen Gegenden der Alten Welt Erinnerungskulturen, die sich an Glanzzeiten der Vergangenheit orientierten. Im Ägypten der 25. und 26. Dynastie entwickelt sich gegen Ende des 8. Jahrhunderts v.Chr. ein ausgeprägter Archaismus, der alle Gebiete der Kultur ergreift. Alle Epochen der Vergangenheit, vom Alten Reich (3.Jahrtausend) bis zur 18. Dynastie (1400 v.Chr.), besonders aber die Zeit des Mittleren Reichs, gelten jetzt als die maßgeblichen Vorbilder. ${ }^{7}$ Ein Grabherr aus dem 7. Jahrhundert v.Chr. besucht in Mittelägypten das anderthalb Jahrtausende ältere Grab eines Namensvetters und kopiert daraus eine Wand in seinem eigenen Grab. ${ }^{8}$ In einem Papyrus aus der Römerzeit finden sich die Kopien von Grabfassaden, die aus dem Ende des 3.Jt. stammen. ${ }^{9}$ Die Kultur wird sich ihrer Zeittiefe bewußt und entwikkelt zugleich Vorstellungen einer kulturellen Gleichzeitigkeit, die es möglich machen, sich mit den Ausdrucksformen einer Jahrtausende zurückliegenden Vergangenheit zu identifizieren, so daß es für uns oft schwierig ist, die Texte und Kunstwerke der Spätzeit richtig zu datieren.

((18)) Eine andere, weniger eklektische Form von Vergangenheitsbezug und Erinnerungskultur entwickelt sich im spätzeitlichen Mesopotamien. ${ }^{10}$ Während die Ägypter sich ihre Vorbilder aus verschiedenen Epochen der Vergangenheit zusammensuchen, orientieren sich die assyrischen und babylonischen Könige an der Zeit der Sargonidendynastie (23342154), die in der Rückerinnerung der Spätzeit zur normativen Vergangenheit erhoben wird, aus der alles Handeln seine Legitimation schöpft. Im Bezug auf diese Vergangenheit hat die mesopotamische Kultur seit jeher ihre Krisen überdauert: den Untergang der sumerischen Kultur, die verschiedenen Einwanderungswellen und Fremdherrschaften, die schweren Konflikte zwischen Assyrern und Babyloniern und die persische Eroberung. Im 1. Jt. v.Chr., als die Ruinen der Sargonidenzeit so gut wie verschwunden waren, verwandelte sich die mesopotamische Gesellschaft in eine "digging society", die regelrechte Ausgrabungen veranstaltete, um an die Spuren ihrer normativen Vergangenheit heranzukommen. Das kulturelle Gedächtnis nahm die Form einer "topographie légendaire" an, wie das der Soziologe Maurice Halbwachs für das Palästina der spätantiken und frühmittelalterlichen Kirche gezeigt hat. ${ }^{11}$

((19)) Was Griechenland angeht, brauchen wir nur im Vorübergehen an den wohlbekannten Fall der homerischen Epen $\mathrm{zu}$ erinnern. Nicht nur greifen sie über den Abgrund eines 
"dunklen Zeitalters" von vier- bis fünfhundert Jahren auf die späte Bronzezeit zurück und erheben den Trojanischen Krieg zur normativen Vergangenheit, sondern sie gewinnen im 6. und 5. Jahrhundert, insbesondere die Ilias, auch ihrerseits den Rang einer zentralen, identitäts-fundierenden und in diesem Sinne "konnektiven" Erinnerung. Es ist die staatenübergreifende Identität eines panhellenischen Zusammengehörigkeitsbewußtseins, das sich in dieser Geschichte einer panhellenischen Koalition gegen einen Feind im Osten spiegelt, bestärkt und in jeder Rezitation festlich erneuert. ${ }^{12}$

((20)) Zweifellos ist in diesen frühen Phänomenen eines schriftgestützten, in tiefe Erinnerungsräume zurückgreifenden kulturellen Gedächtnisses auch viel Erinnerungspolitik im Dienste des "kollektiven Gedächtnisses" (im Sinne von Aleida Assmann, s. dort) am Werk. Dennoch sprengen jetzt im Medium der Schrift die Erinnerungsbestände schnell den Horizont des unmittelbar gebrauchten Vergangenheitswissens und überformen das "Bindungsgedächtnis" durch ein viel weiter ausgreifendes "Bildungsgedächtnis". Die normativen Texte, wie in Israel die Tora, in Ägypten das Totenbuch, in Mesopotamien das Enuma Elisch und das Gilgamesch-Epos, in Griechenland die homerischen Epen, bilden einen Kern, um den sich ganze Bibliotheken bilden. Die Palastbibliothek von Ninive ist das früheste Beispiel einer umfassenden Bildungsbibliothek, die das ganze Wissen aus Gegenwart und Vergangenheit versammeln will und deren berühmtestes Beispiel dann 500 Jahre später die Bibliothek von Alexandria darstellt. Das kulturelle Gedächtnis des multikulturellen Hellenismus ist komplex, pluralistisch, synkretistisch, es umgreift eine Menge von in Zeit und Raum verschiedenen Bindungsgedächtnissen und WirIdentitäten und bezieht aus diesen Spannungen und Widersprüchen seine Dynamik.

((21)) Das kulturelle Gedächtnis hat seinerseits einen Außenhorizont von Wissensbeständen, in Bezug auf die sich der Begriff des "Gedächtnisses" verbietet. Von daher gesehen zeigt sich der Gedächtnisaspekt, die Gedächtnisförmigkeit der Kultur. Es gibt vieles, was außerhalb des kulturellen Gedächtnisses liegt und was uns dann nur noch als vergangen, aber nicht mehr in dem oben beschriebenen Sinne zugleich auch als gleichzeitig anmutet. Es gibt da eine zwar bewegliche, aber doch sehr deutliche Grenze zwischen innen und außen.

((22)) Dafür liefert die 3000-Jahrfeier der Stadt Jerusalem im Jahre 1996 ein besonders schlagendes Beispiel. Das Datum ergibt sich, weil David die Stadt vor 3000 Jahren gegründet haben soll. Nun steht zwar in der Bibel, daß er sie nicht gegründet, sondern von den Jebusitern erobert hat, aber die jebusitische oder kanaanäische Vorgeschichte Jerusalems wird als so unbedeutend abgetan, daß man von Davids Eroberung als der eigentlichen Gründung ausgehen zu können glaubt. Dem Historiker allerdings stellen sich die Dinge ganz anders dar. Jerusalem geht als Stadt um weitere 800 Jahre bis in die mittlere Bronzezeit zurück. Schon damals war sie mit einer Stadtmauer befestigt, umfaßte eine Fläche von ca. $4 \mathrm{~h}$ und zählte ca 2500 Einwohner. Nach damaligen Maßstäben handelte es sich um eine richtige, keineswegs unbedeutende Stadt. In der späten Bronzezeit, also ca. 350 Jahre vor dem angeblichen Gründungsdatum, war Jerusalem ein Stadtstaat namens
Uru-salima mit einem König und einer militärischen und zivilen Verwaltungsbürokratie. Er trieb Handel und unterhielt diplomatische Beziehungen zu den Großmächten der damaligen Zeit, besonders Ägypten, wo sich 6 Briefe des Königs Abdicheba erhalten haben. Das alles ist lange bekannt. Aber es ist nur "Alte Geschichte", nicht bewohnte Vergangenheit. Niemand von den heute in Jerusalem lebenden und um Jerusalem streitenden Gruppen, Juden, Araber, Christen, nimmt die kanaanäische Vergangenheit Jerusalems als die eigene in Anspruch. Das interessiert nur die Historiker. Die 800jährige kanaanäische Vorgeschichte Jerusalems, mit anderen Worten, liegt außerhalb des Horizonts kultureller Erinnerung, der sich also in diesem Falle einmal auf 3000 Jahre beziffern läßt.

((23)) Von 3000 Jahren spricht auch Goethe in jenen Versen aus dem West-Östlichen Diwan:

Wer nicht von 3000 Jahren
Sich weiß Rechenschaft zu geben
Bleib im Dunkeln unerfahren
Mag von Tag zu Tage leben. ${ }^{13}$ -

((24)) Auch Goethe wußte, daß die Geschichte viel weiter zurückreicht. Zu seiner Zeit rechnete man gelegentlich noch nach "anno mundi", so z.B. Vico, bei dem der Trojanische Krieg im Jahre 2800 A.M., also nach damaliger Chronologie ziemlich exakt 3000 Jahre vor Abfassung des West-Östlichen Diwans stattfand. Für Goethe beginnt, nicht die Menschheitsgeschichte, sondern das kulturelle Gedächtnis des Abendlandes mit der Zeit, in der man traditionell den Trojanischen Krieg und den Exodus aus Ägypten ansetzte. Diese beiden Ereignisse bildeten die Grenzmarken des europäischen Kulturgedächtnisses. Beides waren Akte der Auswanderung, der Ablösung vom Orient. Die Israeliten wanderten aus Ägypten aus und Aeneas aus Troja in Kleinasien. Denn selbstverständlich blickte man das ganze Mittelalter und bis weit ins 18 . Jahrhundert hinein auf den Trojanischen Krieg durch die Brille der Aeneis und nicht der Ilias. Moses und Aeneas, die beiden Auswanderer, waren die Gründungsheroen des Abendlandes.

\section{Kulturelle Texte in Mündlichkeit und Schriftlichkeit}

((25)) Das "Kulturelle Gedächtnis" ist daher nicht gleichbedeutend mit Geschichte und Geschichtsbewußtsein, sondern eine eigenständige Form des Vergangenheitsbezugs. Es ist gekennzeichnet durch Horizont und Prägnanz. Nicht die irgend auffindbaren Quellen und Spuren, sondern die kulturellen Texte bestimmen die Reichweite seines Horizonts und geben ihm durch die weltmodellierende Funktion ihrer Semantik seine identitätsfundierende Prägnanz. Kulturelle Texte sind daher alle semantischen oder symbolischen Artikulationen, die in einer gegebenen Gesellschaft im Rahmen "zerdehnter Situationen" kommuniziert werden; dazu gehören über das Sprachliche hinaus auch Bilder, Gesten, Tänze, Riten, Sitten, Bräuche, Baudenkmäler, Städtebilder, ja sogar Landschaften wie die australischen "song-lines", solange sie nur als semantische und nicht lediglich geographische Einheiten verstanden und im Leben der Gemeinschaft im Sinne der "wiederaufgenommenen Botschaft" reproduziert 
und reaktualisiert werden. Diese Texte sind ver-bindlich im doppelten Sinne der Bindung, als verpflichtende und als verbindende Strukturen. Sie üben ihre verbindende Wirkung auf die Gruppe im normativen und im formativen Sinne aus. Normative kulturelle Texte formulieren die Regeln des Zusammenlebens und reichen von Sprichwörtern über Erziehungsbücher und Verhaltenslehren bis $\mathrm{zu}$ kanonischen und klassischen Texten wie der Torah, dem Schulchan Aruch und der Nikomachischen Ethik. Formative Texte formulieren das Selbstbild der Gruppe und reichen von Stammesmythen und Ursprungssagen bis zu literarischen Werken wie Homer und Vergil, Dante, Shakespeare, Milton und Goethe. Durch die Reproduktion kultureller Texte im Rahmen "zerdehnter Situationen" reproduziert sich eine Gesellschaft oder Kultur selbst durch die Abfolge der Generationen hindurch in identischer oder zumindest wiedererkennbarer Form.

((26)) Innerhalb dieses generellen Funktionsrahmens des kulturellen Gedächtnisses, in dem mündliche und schriftliche, sprachliche und außersprachliche Kommunikation gleichermaßen als kulturelle Texte fungieren, bedeutet die Einführung der Schrift dann einen grundsätzlichen und tiefgreifenden Wandel. Das betrifft zum einen die Zeitstruktur der "zerdehnten Situation" und zum anderen, was man die Raumstruktur des kulturellen Gedächtnisses nennen könnte, das sich mit zunehmender Schriftlichkeit in Vorder- und Hintergrund, zugängliche, entlegene und unzugängliche Bereiche aufteilt.

((27)) Alle schriftlosen Institutionalisierungen der zerdehnten Situation hängen von Zeit und Raum ab. Man muß auf die Wiederkehr eines Festes und einer Begehung warten oder zu einem Bild, einem Monument, einem heiligen Ort pilgern, um an der Kommunikation der Botschaft teilzuhaben. Das ist bei schriftlichen Texten anders: man braucht sie nur zu lesen, jederzeit, überall. Dazu bedarf es nur zweier Voraussetzungen: daß die Texte zur Hand und daß sie lesbar sind. Die erste Voraussetzung läßt sich verhältnismäßig leicht erfüllen; die zweite ist wesentlich anspruchsvoller. Schriftliche Kommunikation kultureller Texte funktioniert nur im Rahmen intensivster institutioneller Absicherungen. Damit die Texte durch den Wandel der Zeiten und Geschlechter hindurch ihre normativen und formativen Impulse freisetzen können, müssen sie fortwährend den sich wandelnden Verstehensrahmen angepaßt werden. Das geschieht anfänglich durch Ersetzung, Erweiterung, Umschreibung, Fortschreibung: so wandelt sich etwa das Gilgamesch-Epos im Laufe seiner jahrtausendelangen Überlieferungsgeschichte von einem mehr oder weniger lockeren Sagenzyklus zu einem streng komponierten 12-Tafel-Gedicht ${ }^{14}$ und das Buch Jesaja erweitert sich um Deuteround Tritojesaja. ${ }^{15}$ Die Texte sind in Umfang und Aufbau nicht festgelegt, sondern weisen ein Ausmaß von Fluktuation auf, das der mündlichen Überlieferungsform noch nahesteht. Der Mediävist Paul Zumthor prägte dafür den Begriff "mouvance" ${ }^{\prime 16}$ und A. Assmann beschrieb diese Überlieferungsform als "schriftliche Folklore". 17

((28)) Dieser mouvance oder Flexibilität wird mit dem Vorgang der Kanonisierung ein kategorisches Ende gesetzt. Jetzt wird der kulturelle Text in seinem Wortlaut so strikt festgelegt, wie es bis dahin nur für heilige Texte im Rahmen ritueller Kommunikation üblich war. Der Unterschied zwischen heiligen und kulturellen Texten liegt darin, daß bei ersteren alles auf die korrekte Rezitation, bei letzteren alles auf den inneren Nachvollzug ankommt. Heilige Texte müssen nicht verstanden werden. Sie vergegenwärtigen im Akt ihrer Rezitation das Heilige und wirken wie ein sprachlicher Tempel. Kulturelle Texte dagegen vermitteln ihre normativen und formativen Impulse nur im Akt des Verstehens. Daher müssen sie durch Glossen, Erweiterungen, Umschreibungen transparent gehalten werden. Im Akt der Kanonisierung werden die kulturellen Texte wie heilige Texte behandelt. Nun darf nichts mehr an ihrem Wortlaut verändert, hinzugefügt oder weggenommen werden. ${ }^{18}$ Damit werden sie so dunkel wie heilige Texte und ohne das Hinzutreten eines Auslegers unverständlich. ${ }^{19}$

((29)) So beobachten wir in der Alten Welt gleichzeitig mit der Wortlautfixierung der kulturellen Texte, zuerst in Babylonien und dann in Israel und Alexandria, das Aufblühen einer Auslegungskultur. Kommentare werden geschrieben, Auslegungsregeln (Hermeneutik) festgelegt, Lehrhäuser gegründet, in denen die kanonisierten kulturellen Texte ausgelegt werden, und der gelehrte Schreiber rückt zum geistigen Führer auf. Das Schwergewicht der Vermittlung und Partizipation am kulturellen Sinn verlagert sich von den Riten und Festen in die Lehrhäuser und Wortgottesdienste.

((30)) Sowohl auf Seiten der mündlichen als auch der schriftlichen Überlieferung weisen die Institutionen einen hohen Grad an Spezialisierung auf. Auf der mündlichen Seite fungieren die Gedächtnisspezialisten als Medien sowohl der Speicherung als auch der Überlieferung und Aufführung. Sie müssen die heiligen und kulturellen Texte memorieren, ihren Lehrlingen und Gehilfen beibringen und in den entsprechenden Aufführungssituationen entweder wörtlich oder in kreativer Anpassung an das Publikum realisieren. Sie sind sowohl Memoralisten als auch Ritualisten. ${ }^{20}$

((31)) Auf der Seite der Schriftlichkeit werden die mit der Überlieferung der kulturellen Texte betrauten Schreiber zu Philologen und Kommentatoren, Lehrern und Predigern, deren Aufgabe nicht nur darin besteht, die Texte zu kopieren, sondern sie in wechselnde Lebenswirklichkeiten zu übersetzen, indem sie ihre normativen und formativen Impulse freisetzen.

((32)) Im Bereich der Mündlichkeit bedarf die Institutionalisierung der zerdehnten Situation eines erheblich höheren Grades an Formalisierung als im Bereich der Schriftlichkeit. Dadurch erklärt sich erstens der viel höhere Grad an Professionalisierung auf Seiten der Gedächtnisträger und zweitens die Fülle und Bedeutung der Riten in schriftlosen Gesellschaften. In frühen Schriftkulturen wie Mesopotamien und Ägypten gibt es keine hauptberuflichen Lehrer und Ausleger; diese Funktionen werden von verschiedenen Schreiber-Beamten im Rahmen ihrer sonstigen Berufstätigkeiten miterfüllt. Die professionelle Ausdifferenzierung von Spezialisten in der Überlieferung kultureller Texte beginnt erst mit der Kanonisierung. In Gedächtniskulturen dagegen sind die Ritualisten-Memoralisten klar von der übrigen Gesellschaft abgegrenzt: Schamanen, Medizinmänner, Griots, Barden usw. widmen sich in der Regel ganz ihrer Aufgabe als Überlieferungsträger der heili- 
gen und kulturellen Texte. Bereits der jüdische Historiker Josephus Flavius bringt im 1.Jh.n.Chr. den Unterschied zwischen Gedächtniskultur und Buchkultur oder "ritueller" und “textueller Kohärenz" auf den Punkt, wenn er Judentum und Hellenismus gegenüberstellt:

Wo wäre demnach eine gleich ehrwürdige Staatsverwaltung zu finden? Wo eine, die mit der Ehrfurcht gegen Gott in schönerem Einklang stände? Wenn alle Schichten des Volkes zur Frömmigkeit erzogen werden, wenn die Pflege der letzteren vornehmlich den Priestern anvertraut ist - sieht das nicht aus, als ob das gesamte öffentliche Leben eine einzige heilige Festfeier wäre? Was die Heiden unter dem Namen Mysterien und Weihen nur in wenigen Tagen begehen, ohne es jedoch dauernd in ihren Herzen bewahren zu können, daran halten wir mit unendlichem Entzücken und unverrückten Sinnes allezeit fest. ${ }^{21}$

Die Heiden müssen warten bis zur nächsten Durchführung des Rituals, aber die Juden sind im ständigen Besitz ihrer kulturellen Texte, weil sie in "öffentlichem Unterricht" von den Priestern darin unterwiesen werden. Ihre "Mysterien" sind permanent und kontinuierlich. Sie bestehen in der von priesterlicher Auslegung geleiteten Lektüre der heiligen und kulturellen Texte.

((33)) Dieser Fall läßt sich verallgemeinern. Kulturelle Texte bilden das diachrone Rückgrat einer Gesellschaft, das ihre Identität und Kohärenz durch die Abfolge der Generationen sicherstellt. Das Prinzip dieser Kohärenz kann entweder rituell oder textuell realisiert sein. Rituelle Kohärenz bildet dort das Prinzip kultureller Reproduktion, wo die kulturellen Texte in der Form kommuniziert werden, die Josephus den Heiden zuschreibt: in Festen und Riten. Textuelle Kohärenz herrscht vor, wo die kulturellen Texte in Form von Lehren und Lernen, Lesen und Auslegen kommuniziert werden, wie es Josephus von den Juden berichtet. Je mehr eine Gesellschaft durch Schrift bestimmt ist, desto weniger spielen die Riten in ihr eine Rolle. Den entscheidenden Wandel in dieser Hinsicht hat nicht die Erfindung der Schrift, sondern der Buchdruck herbeigeführt. ${ }^{22}$

\section{Randbezirke des kulturellen Gedächtnisses: Speicher, Archiv und Krypta}

((34)) Ebenso bedeutsam wie die Wandlungen in der Zeitstruktur der zerdehnten Situation sind die Wandlungen in der Raumstruktur des kulturellen Gedächtnisses. Für schriftlose Gesellschaften gilt, daß nur das im kulturellen Gedächtnis lebendig bleibt, was jeweils gebraucht wird. Horizont und Prägnanz des kulturellen Gedächtnisses bestimmen sich immer neu nach den Gegebenheiten und Erfordernissen einer jeweiligen Gegenwart. In Schriftkulturen dagegen wächst überlieferter, in symboliSche Formen ausgelagerter Sinn zu riesigen Archiven an, von denen nur mehr oder weniger beschränkte zentrale Teilbereiche in einer gegebenen Gegenwart wirklich gebraucht, bewohnt und bewirtschaftet werden, während sich darum herum Bereiche des nicht mehr Gebrauchten ablagern, die im Grenzfall dem vollkommenen Verschwinden und Vergessen gleichkommen. Aleida Assmann hat daher den Vorschlag gemacht, Zwischen Funktions- und Speichergedächtnis zu unterscheiden. ${ }^{23}$ Der Begriff des Speichergedächtnisses eröffnet Möglichkeiten in Richtung kultureller Formen von Unbewußtheit. Die Kultur ist ein Palimpsest und gleicht auch darin dem individuellen Gedächtnis, für das ja Sigmund Freud die Stadt Rom als eine Lieblingsmetapher verwendete. Rom besteht eben gerade nicht nur aus einem gewaltigen Freilichtmuseum, in dem die Vergangenheit konserviert und ausgestellt ist, sondern aus einem unentwirrbaren Ineinander von Altem und Neuem, Verbautem und Verschüttetem, Wiederverwendetem und Ausgesondertem. So entstehen Spannungen, Verwerfungen, Antagonismen zwischen dem Zensierten und dem Unzensierten, dem Kanonischen und dem Apokryphen, dem Orthodoxen und dem Häretischen, dem Zentralen und dem Marginalen, die für kulturelle Dynamik sorgen. Die Grenze zwischen dem Speicher- und dem Funktionsgedächtnis verschiebt sich ständig. Darin sieht Aleida Assmann, ,die Bedingung der Möglichkeit von Veränderung und Erneuerung“. ${ }^{24}$ Deshalb ist auch der Begriff der Tradition, zumindest im herkömmlichen Sinne, für dieses Phänomen völlig unzureichend. Tradition meint das Geschäft des Überlieferns und Rezipierens sowie den Bestand des Überlieferten. Mit diesem Begriff ist keine andere Dynamik denkbar als die in solcher kulturellen Arbeit bewußt und kontrolliert entfaltete und jede Interaktion mit der Dynamik von Identität und Erinnerung ist abgeschnitten. Der Begriff der Tradition läßt keinen Raum für das Unbewußte.

((35)) Nun geht es sicher zu weit, das Speichergedächtnis einfach mit dem kulturellen Unbewußten gleichzusetzen. Das Speichergedächtnis ist entgrenzt und amorph, die strukturierenden, form- und horizontbildenden Prinzipien der Funktion sind hier weggefallen, wie sie aus den Bedürfnissen der Gruppe nach Identität, Normativität und Orientierung erwachsen. Damit sind diese Bestände nur an die Peripherie verschoben, aber nicht prinzipiell unzugänglich, verdrängt, verbannt oder sonstwie unverfügbar geworden. Aber das gibt es offenbar auch. Daher ist es nötig, auch auf der Ebene des kulturellen Gedächtnisses, und zwar des Speichergedächtnisses, nach Analogien zu dem Ausschau zu halten, was Freud auf der Ebene des individuellen Gedächtnisses als Verdrängung bezeichnet hat. Gerade in seiner Verdrängungslehre hat Freud ja in einer bis heute äußerst umstrittenen Weise Massenpsychologie und Individualpsychologie verkoppelt. Der Historiker Yosef Hayim Yerushalmi hatte das Problem der religiösen Tradition, das Freud als Wiederkehr des Verdrängten deutet, in den Mittelpunkt seiner Freud-Interpretation gestellt und auf die Unterscheidung von biologisch-genetischer Vererbung und bewußter kultureller Weitergabe zugespitzt. Im Licht dieser Zwangsalternative schlug er dann Freuds Thesen der Seite der biologischen Vererbung zu. ${ }^{25}$ Das betraf nicht nur die phylogenetischen Erinnerungsspuren aus der "Urhorde", als der Vater von seinen Söhnen, die er mit Kastration bedrohte, erschlagen wurde, die sich in der menschlichen Seele zur "archaischen Erbschaft" verdichteten und die ödipalen Konflikte produzieren, sondern es betraf auch Freuds These von dem Mord an Mose, der speziell die jüdische Seele traumatisiert, aber auch auf dem Wege der Wiederkehr des Verdrängten zur Aufnahme und Bewahrung des Monotheismus disponiert haben soll. Das weist Yerushalmi als "Psycho-Lamarckismus" zurück. Jean Baptiste de Monet de Lamarck hatte zu Anfang des 19. Jahrhunderts die Vererbung erworbener Eigenschaften behauptet und dadurch nicht wenig zur Entstehung von Rassentheorien beigetragen.

((36)) Gegen Yerushalmis Vorwurf des Psycho-Lamarckismus 
hatte Jacques Derrida in einem vierstündigen, am 5. Juni 1994 in London gehaltenen Vortrag Freud verteidigt, indem er die Zwangsalternative von genetischer Vererbung und bewußter Überlieferung aufbrach und ein Drittes einführte, das er "Archiv" nannte. ${ }^{26}$ Darauf aufbauend entwickelte Richard J. Bern$\operatorname{stein}^{27}$ einen erweiterten Begriff von Tradition, der unbewußte Aspekte der Weitergabe und transgenerationellen Übertragung einschließt. In Freuds Augen wird der herkömmliche Traditionsbegriff der Dynamik religiöser Traditionen nicht gerecht (Freud versteht unter "Tradition" mündliche Überlieferung, die er der schriftlichen Geschichtsschreibung gegenüberstellt). Diese Dynamik wird gekennzeichnet durch Brüche, Diskontinuitäten, Verschüttungen, Wiederkünfte, Durchbrüche usw., die sich niemals allein auf das Geschäft bewußter Überlieferung zurückführen lassen, und der nur der Vergleich mit den Phasen einer individuellen Neurose gerecht wird: Frühes Trauma - Abwehr und Verdrängung - Latenz - Ausbruch der neurotischen Erkrankung - teilweise Wiederkehr des Verdrängten. Die Religionsgeschichte verläuft in ähnlichen Wellen. Bernsteins erweiterter Traditionsbegriff, der Freuds Kritik aufnimmt und unbewußten Übertragungen Rechnung trägt, greift auf den "ontologischen" Traditionsbegriff Hans Georg Gadamers zurück. Die Position Gadamers bezeichnet für Bernstein den kulturalistischen Gegenpol zur biologistischen Position Lamarcks. Gadamer hat in Wahrheit und Methode (1960) Heideggers Vorstellungen von der sprachlichen Verfaßtheit der menschlichen Existenz weitergedacht in Richtung "Text", d.h. inhaltlich bestimmter, sprachlich artikulierter und befestigter Traditionen, die jede Gegenwart konstituieren, indem sie das Vorverständnis fundieren, aus dem jedes Verstehen gespeist ist. Kein Verstehen ohne Erinnerung, kein Dasein ohne Tradition. Derrida, dessen Denken sich ja ebenfalls aus Heideggerschen Ansätzen speist, hat den $\mathrm{Be}$ griff des "Archivs" in ganz entsprechender Weise ausgeleuchtet als eine gegenwartskonstituierende und zukunftsermöglichende Gedächtnisform im Medium sprachlicher und außersprachlicher, diskursiver und nichtdiskursiver Symbole und darin geht er am Leitfaden etymologischer Assoziationen des Wortes "Archiv" (arché, archeion, Archonten, Patri-Archiv, Matri-Archiv usw.) über Gadamer hinaus - durchwaltet von politischen Strukturen der Macht und der Herrschaft.

((37)) Der Begriff des kulturellen Gedächtnisses entspricht dem, was Derrida "Archiv" und Bernstein "Tradition" nennen, und weiß sich ebenso wie sie den Freudschen Einsichten in die psychohistorische Dimension und Dynamik der kulturellen Überlieferung verpflichtet. Das kulturelle Gedächtnis umfaßt im Gegensatz zum kommunikativen Gedächtnis das Uralte, Abgelegene, Ausgelagerte und im Gegensatz zum kollektiven oder Bindungsgedächtnis das Nichtinstrumentalisierbare, Häretische, Subversive, Abgespaltene.

((38)) Nicht von ungefähr hat sich diese Debatte um die Möglichkeit unbewußter kultureller Übertragungen an Freuds Moses-Buch entzündet. ${ }^{28}$ Die Entstehung der monotheistischen Religion ist in der Tat der klassische Fall für einen Vorgang, den man in Weiterführung der Freudschen Analyse als "kulturelle Kryptenbildung" bezeichnen möchte. Mit der Durchsetzung des Monotheismus wurden sowohl die anderen Religionen als auch und vor allem die eigene Tradition als Heidentum ausgegrenzt. Damit wurden weite Bereiche der kulturellen Überlieferung unterdrückt, marginalisiert, in subkulturelle Folklore abgeschoben oder geradezu kriminalisiert. Das vom Monotheismus geprägte kulturelle Gedächtnis wurde komplex; zum Entlegenen, Nichtgebrauchten, Veralteten ("Speicher") trat das Apokryphe, Ausgegrenzte und Verbotene ("Krypta"), das gleichwohl in der Form des Verbots als Gegengedächtnis erhalten blieb, so daß etwa Gottfried Arnold seine "Unpartheyische Ketzergeschichte" schreiben und in Goethe einen begeisterten Leser finden konnte. Das Heidentum verschwand nie aus dem kulturellen Gedächtnis des Abendlandes ${ }^{29}$ und insbesondere das durch zahllose griechische und lateinische Texte vermittelte Ägypten spielte darin eine ambivalente und höchst virulente Rolle. ${ }^{30}$

((39)) Mit der revolutionären Entwicklung der Elektronik und des Internet als neue Speicher- und Kommunikationsmedien bahnen sich tiefgreifende Veränderungen in der Architektur des kulturellen Gedächtnisses an. Darüber können wir einstweilen nur Vermutungen anstellen. Sie werden vor allem zwei Grenzen betreffen, die sich verschieben und verwischen werden: die Grenze zwischen dem kommunikativen und dem kulturellen Gedächtnis und die Grenzen zwischen Funktions- und Speichergedächtnis, Archiv und Krypta. Im virtuellen Raum des Internet läßt sich, anders als in der realen Welt, zugleich schriftlich und interaktiv, weltweit und zeitgleich kommunizieren. Hier entstehen, anders als in der normalen Kommunikation, Texte, die als wiederabrufbare Bestände (zumindest für eine gewisse Zeit) zurückbleiben, wobei fraglich ist, in welchem Umfang der Textbegriff hier überhaupt noch anwendbar ist. Dadurch verschwinden charakteristische Unterscheidungsmerkmale zwischen dem kommunikativen und dem kulturellen Gedächtnis. Auch das Unbedeutendste und Ephemerste gerinnt zum Text, auch der Text zerfließt in vergleichsweise amorphe, jeder Um- und Neugestaltung offenstehende Datenmengen.

((40)) Durch die exponentiell gesteigerten Speicherungsmöglichkeiten des Computers werden Grenzen und Selektionsmechanismen hinfällig, die von der Ökonomie und Verwaltbarkeit materieller Speichermedien diktiert sind. Die Masse des Speicherbaren wächst ins Uferlose und führt zu einer entsprechenden Auflösung der traditionellen Horizontbildungen. Gleichzeitig halten immer leistungsfähigere Suchmaschinen mit dieser Entwicklung Schritt und ermöglichen die jederzeitige Verfügbarkeit auch der scheinbar entlegensten Informationen. Somit verschwinden die gesellschaftlich vorgegebenen, wertbezogenen Relevanzperspektiven, die das Funktionsgedächtnis gegenüber dem Speichergedächtnis hervorheben, das Wichtige vom Unwichtigen absetzen und Struktur und Orientierung in das Labyrinth der kulturellen Erinnerung bringen. Die Suchmaschinen helfen dem Individuum, sich seinen je eigenen Weg durch den Informations-Dschungel zu bahnen und seine individuellen Relevanzperspektiven auszubilden. Zentrum und Peripherie bestimmen sich nicht mehr nach sozial und kulturell vorgegebenen Kriterien und Filtern, sondern nach Neugier, Interesse und Geschmack des jeweiligen "users".

((41)) Das Internet fördert die beiden gegenläufigen Prozesse der Globalisierung und der Regionalisierung. Zugleich mit der Erschließung eines globalen Kommunikationsraums bilden 
sich darin Mikrohorizonte der Kommunikation und Erinnerung, sog. chat-rooms, aus, in denen im Medium interaktiven Datenaustauschs Interessengruppen zusammenfinden und für einige Zeit so etwas wie ein eigenes Gruppengedächtnis ausbilden können. Auch der Zusammenhang von Kommunikation, Gedächtnis und Identität verändert sich. Die Teilnehmer solcher Kommunikationsformen bedienen sich meist einer virtuellen, frei konstruierten Identität, die als Maske oder "Avatar" in Konkurrenz zu ihrer realen Identität tritt, wobei aber die Maske jederzeit fallen gelassen und die Realidentität ins Spiel gebracht werden kann.

((42)) Das Internet bietet eine neue Institutionalisierung der "zerdehnten Situation", in der nicht mehr die Zeit, sondern der Raum privilegiert wird. Bisher betraf die "Zerdehnung" in allererster Linie die Zeit; die entscheidenden Motive in der Ausbildung eines kulturellen Gedächtnisses waren die Stabilisierung von Erfahrung und die Erschließung lebenszeittranszendierender Zeithorizonte. Das Internet ermöglicht demgegenüber die Transzendierung der räumlichen Beschränkungen; es verheißt nicht Unsterblichkeit, aber weltweite Kommunikation. Die Zeit wird nicht "zerdehnt", sondern im Gegenteil zum Verschwinden gebracht, indem die schriftgestützte Fernkommunikation wieder auf die "Echtzeit" mündlicher Interaktion reduziert wird.

((43)) Im Zeichen der Kommunizierbarkeit, Speicherbarkeit und Auffindbarkeit von schlechthin allem und jedem kann es die Entstehung von Nischen und Krypten im kulturellen Gedächtnis und damit die Ausbildung eines kulturellen Unbewußten eigentlich nicht mehr geben. Der exotischste Kult, die satanischste Häresie, die abwegigste Spezialinformation stehen gleichrangig neben dem, was bisher als wichtig, heilig oder klassisch unbedingten Vorrang genoß. Das Internet läßt keine Verdrängung zu.

((44)) Was diese Veränderungen für die Zukunft des kulturellen Gedächtnisses bedeuten, ist noch kaum absehbar, zumal sich die virtuelle Welt bislang nur neben, aber nicht an die Stelle der traditionellen Realwelt setzt, deren Strukturen kaum beeinflußt daneben weiterbestehen.

\section{Anmerkungen}

1 George Kubler, The Shape of Time. Remarks on the History of Things, New Haven 1962; dt. Die Form der Zeit, Frankfurt 1982

2 Zu Riten als mnemotechnischen Verfahren s. Carlo Severi, La memoria rituale. Follia e immagine del Bianco in una tradizione sciamanica amerindia, Florenz 1993.

3 N. Luhmann, Gesellschaftsstruktur und Semantik I, Frankfurt 1980, 19-21, mit Verweis auf E. Havelock, Preface to Plato, Cambridge Mass. 1963.

4 P. Hartmann, „Zum Begriff des sprachlichen Zeichens“, in: Zeitschrift für Phonetik, Sprachwissenschaft und Kommunikationsforschung 21, 1968, 205-22 (Zitat: 212)

5 K. Ehlich, „Text und sprachliches Handeln. Die Entstehung von Texten aus dem Bedürfnis nach Überlieferung“, in: A. u. J. Assmann, Chr. Hardmeier (Hrsg.), Schrift und Gedächtnis, München 1983, 24-43.

6 Siehe Ehlich, a.a.O., 30f.

7 Peter Der Manuelian, Living in the Past. Studies in the Archaism of the Egyptian Twenty-Sixth Dynasty, London 1994.

8 W. Schenkel, K. Kuhlmann, Das Grab des Ibi. Theben, Nr. 36, Band I, Mainz 1983, 89-106; Der Manuelian, a.a.O., 24-28.
9 J. Osing, G. Rosati, Papiri Geroglifici e ieratici da Tebtynis, Firenze 1998, 55-91

10 Gerdien Jonker, The Topography of Remembrance. The Dead, Tradition \& Collective Memory in Mesopotamia, Leiden 1995.

11 Maurice Halbwachs, La topographie legendaire des évangiles en Terre Sainte, Paris 1941

12 Vgl. hierzu Jan Assmann, Das kulturelle Gedächtnis. Schrift, Erinnerung und politische Identität in frühen Hochkulturen, München 1992, 2. Aufl. 1997, (Taschenbuchausgabe München 1998), Kap. VII, sowie ders., Religion und kulturelles Gedächtnis. Zehn Studien, München 2000.

13 J. W. Goethe, West-Östlicher Diwan, Buch des Unmuts.

14 A. George, The Epic of Gilgamesh. The Babylonian Epic Poem and Other Texts in Akkadian and Sumerian, New York 1999.

15 O. H. Steck, Der Abschluß der Prophetie im Alten Testament. Ein Versuch zur Vorgeschichte des Kanons, Biblisch Theologische Studien 17, Neukirchen-Vluyn 1991

16 P. Zumthor, Introduction à la poesie orale, Paris 1983, 245-261.

17 S. Aleida Assmann, „Schriftliche Folklore. Zur Entstehung und Funktion eines Überlieferungstyps“, in: A. u. J. Assmann, C.Hardmeier (Hrsg.), Schrift und Gedächtnis, München 1983, 175-193.

18 G. Offner, „A propos de la sauvegarde des tablettes en AssyroBabylonie“, Revue d'Assyriologie et d'Archéologie 44 (1950) 135-43. Die Kanonformel "Nichts hinzufügen, nichts wegnehmen, nichts verändern" findet sich bereits im Kolophon des Erra-Epos, s. M. Fishbane, „Varia Deuteronomica“, ZAW 84 (1985), 350-52.

19 Zum Zusammenhang von Kanonisierung und Auslegung s. Verf., B. Gladigow (Hgg.), Text und Kommentar, München 1995.

20 Eindrucksvolle Beispiele bei $\mathrm{Ph}$. Borgeaud, „Pour une approche anthropologique de la mémoire religieuse", in: Ders. (Hrsg.), La mémoire des religions, Genf 1988, 7-20.

21 Josephus Flavius, Contra Apionem cap. 22, in: H. Clementz (Hg. und übers.), Josephus Flavius, Kleinere Schriften, Wiesbaden 1993, $177 \mathrm{f}$.

22 S. Elizabeth Eisenstein, The Printing Press as an agent of Change, Communications and cultural transformations in early-modern Europe, Cambridge 1979

23 Aleida Assmann, Erinnerungsräume. Formen und Wandlungen des kulturellen Gedächtnisses, München 1999, 130-145.

24 Erinnerungsräume, 136.

25 Yosef Hayim Yerushalmi, Freud's Moses. Judaism terminable and interminable, New Haven 1991, dt. Freuds Moses. Endliches und unendliches Judentum, Berlin 1992.

26 Jacques Derrida, Mal d'Archive, Editions Galilée, Paris 1995, deutsch 1997: Dem Archiv verschrieben, Brinkmann \& Bose, Berlin 1997.

27 Richard J. Bernstein, Freud and the Legacy of Moses, Cambridge 1998 .

28 S. Freud, Der Mann Moses und die monotheistische Religion, Amsterdam 1939, Verlag Allert de Lange; Gesammelte Werke, Bd. XVI, 101-246; Bibliothek Suhrkamp, Bd. 131, Frankfurt 1964; Kulturtheoretische Schriften, Frankfurt: S.Fischer 1974, 455-581

29 S. vor allem E. Wind, Heidnische Mysterien in der Renaissance, Frankfurt 1981 (orig. Pagan Mysteries in the Renaissance, London 1958); J. Seznik, Das Fortleben der antiken Götter. Die mythologische Tradition im Humanismus und in der Kunst der Renaissance, München 1990 (orig. La survivance des dieux antiques, Paris 1980); C. Ginzburg, Hexensabbat. Entzifferung einer nächtlichen Geschichte, Frankfurt 1993 (or. Storia notturna. Una decifrazione del sabba, Turin 1989).

30 A. Assmann, „Ex Oriente Nox. Ägypten als das kulturelle Unbewußte der abendländischen Tradition“, in: E. Staehelin, B. Jaeger (Hrsg.), Ägypten-Bilder. OBO 1950, Fribourg 1997, 173-186.

\section{Adresse}

Prof. Dr. Jan Assmann, Universität Heidelberg, Ägyptologisches Institut, Marstallhof 4, D-69117 Heidelberg 